\title{
FLOTAÇÃO DE MINÉRIO DE FERRO COM OLEATO DE SÓDIO E SILICATO DE SÓDIO
}

\author{
D. R. NASCIMENTO ${ }^{1}$, T. F. SOUZA ${ }^{2}$ e R. M. F. LIMA ${ }^{2}$ \\ ${ }^{1}$ Instituto Federal de Minas Gerais - Campus de Governador Valadares \\ ${ }^{2}$ Universidade Federal de Ouro Preto \\ debora.nascimento@ifmg.edu.br
}

Artigo submetido em dezembro/2013 e aceito em julho/2014

DOI: $10.15628 /$ holos.2014.1909

\section{RESUMO}

Flotação catiônica inversa de minério de ferro é largamente utilizada no Brasil. Com a contínua diminuição dos teores de ferro nos minérios do Quadrilátero Ferrífero-MG, a flotação direta aniônica dos mesmos seria uma possibilidade a ser considerada. Logo, estudos mais detalhados em termos de reagentes, especialmente depressores, e demais variáveis de processo fazem-se necessários para avaliação da viabilidade de aplicação da mesma para concentrar minérios pobres. Baseado em estudos prévios, esse trabalho teve por objetivo investigar a influência das variáveis (porcentagem de sólidos, dosagem de coletor (oleato de sódio), dosagem de depressor (silicato de sódio) e deslamagem prévia do minério) em $\mathrm{pH} 9$ sobre a flotação de uma amostra de minério de ferro com teores de $\mathrm{Fe}$ e de $\mathrm{SiO}_{2}$ iguais a 35,5 e 52,4\%, respectivamente. Das variáveis estudadas, verificou-se que a deslamagem prévia do minério é de fundamental importância para o processo.

PALAVRAS-CHAVE: Minério de ferro, flotação, oleato de sódio, silicato de sódio, deslamagem.

\section{IRON ORE FLOTATION WITH SODIUM OLEATE AND SODIUM SILICATE}

\begin{abstract}
Cationic inverse flotation of iron ores is frequently applied in Brazil. With the continuing decrease of the iron contents of Quadrilátero Ferrífero-MG iron ore, anionic direct flotation of those ores could be considered. So, detailed studies in terms of reagents, especially depressants, and other process variables must be necessary in order to evaluate the viability to concentrate low grade iron ores with anionic direct flotation. Based in
\end{abstract}

previous studies, this work had as objective to investigate the influence of the variables (solid density, collector dosage (sodium oleate), depressant dosage (sodium metasilicate) and previous ore desliming at $\mathrm{pH} 9$ on the flotation of one iron ore sample with contents of $\mathrm{Fe}$ and $\mathrm{SiO}_{2}$ of 35.5 and $52.4 \%$, respectively. Among studied variables, it was verified that the previous deslimig of ore is of fundamental importance for process.

KEYWORDS: Iron ore, flotation, sodium oleate, sodium silicate, deslimig. 


\section{INTRODUÇÃO}

Devido às diferenças de densidade, de susceptibilidade magnética e de propriedade superficial induzida entre os minerais de ferro e os minerais de ganga, especialmente o quartzo, a concentração de minério de ferro pode ser efetuada por métodos gravíticos, concentração magnética e flotação, que normalmente é aplicada para as frações granulométricas de pellet feed fino $(-0,15 \mathrm{~mm})$. No entanto, a escolha do método de concentração mais adequado para um dado minério depende da composição mineralógica e da malha de liberação (Araújo et al., 2003).

Houot (1983) publicou uma revisão sobre flotação de minério de ferro, destacando a flotação inversa ou reversa dos minerais de ganga (quartzo), usando coletor catiônico (amina) e depressão dos minerais de ferro, usando amido. Na flotação direta de minério de ferro podem ser utilizados coletores aniônicos tais como ácidos graxos saponificados (RCOONa), sulfonatos $\left(\mathrm{RSO}_{3} \mathrm{Na}\right.$ ) e hidroxamatos (RCONHONa), onde $\mathrm{R}$ representa a cadeia de hidrocarbonetos. No entanto, pesquisas de depressores eficazes para flotação direta estão sendo realizadas (Araújo, 2005).

Lopes e Lima (2009) em estudos de microflotação efetuados com amostras de hematita e quartzo, usando oleato de sódio como coletor verificaram que para dosagens a partir de $20 \mathrm{mg} / \mathrm{L}$ foram obtidas flotabilidades do mineral acima de $80 \%$ para todos os valores de $\mathrm{pH}$ testados ( $2 \mathrm{a}$ 12). Para o quartzo, a flotabilidade máxima foi obtida em $\mathrm{pH} 9$ para dosagens de oleato de sódio de 20 e 70 mg/L. Posteriormente, Nascimento et al. (2013) estudaram a influência do silicato de sódio na flotabilidade da hematita e do quartzo para valores de $\mathrm{pH}$ igual a 7 e 9, usando $50 \mathrm{ppm}$ de oleato de sódio como coletor. Nesses estudos verificou-se que a depressão da hematita em $\mathrm{pH}$ 7 foi ligeiramente maior do que em pH 9. O inverso ocorreu para o quartzo. Nesses estudos verificou-se também que o silicato de sódio foi mais eficiente na depressão do quartzo em comparação com a hematita para dosagens menores do que 10 ppm.

Em ensaios de flotação em escala de bancada efetuados com um minério de ferro especularítico, contendo $35,34 \%$ de $\mathrm{Fe}$ e $48,02 \%$ de $\mathrm{SiO}_{2}$ em pH 7, o melhor resultado obtido (recuperação metalúrgica de $\mathrm{Fe}$ igual $88 \%$, teor de Fe no concentrado de 58,1\% e de $\mathrm{SiO}_{2}$ igual a $14,1 \%)$ foi alcançado para polpa com $60 \%$ de sólidos, dosagem de silicato de sódio igual a $600 \mathrm{~g} / \mathrm{t}$ e $1200 \mathrm{~g} / \mathrm{t}$ de oleato de sódio (Lopes e Lima, 2009).

\section{MATERIAIS E MÉTODOS}

Na Tabela 1 está apresentada a análise granuloquímica da amostra de minério de ferro e as composições químicas da amostra deslamada e das lamas.

Pela Tabela 1 observa-se que os teores de todos os compostos analisados, exceto $\mathrm{SiO}_{2}$, da fração granulométrica abaixo de $38 \mu \mathrm{m}$ são maiores do que nas outras faixas granulométricas. Os maiores teores e distribuições de $\mathrm{Al}_{2} \mathrm{O}_{3}$, PPC e menor teor e distribuição de $\mathrm{SiO}_{2}$ na faixa granulométrica abaixo de $38 \mu \mathrm{m}$ estão relacionados com os minerais caolinita e goethita, identificados por microscopia ótica e difratometria de raios X (Lima et al., 2008). Observa-se também, que o teor de $\mathrm{Al}_{2} \mathrm{O}_{3}$ no produto da deslamagem, que representa $11 \%$ em peso, foi de $1,7 \%$, provavelmente devido à remoção dos argilominerais, especialmente caolinita. 
Tabela 1: Análise granuloquímica da amostra de minério de ferro do Quadrilátero Ferrífero.

\begin{tabular}{|c|c|c|c|c|c|c|c|c|c|c|c|}
\hline \multirow{2}{*}{$\begin{array}{c}\text { Tamanho } \\
(\mu \mathrm{m})\end{array}$} & \multirow{2}{*}{$\begin{array}{c}\% \\
\text { Peso }\end{array}$} & \multicolumn{5}{|c|}{ Teores (\%) } & \multicolumn{5}{|c|}{ Distribuição (\%) } \\
\hline & & $\mathrm{Fe}$ & $\mathrm{SiO}_{2}$ & $\mathrm{Al}_{2} \mathrm{O}_{3}$ & $P$ & PPC & $\mathrm{Fe}$ & $\mathrm{SiO}_{2}$ & $\mathrm{Al}_{2} \mathrm{O}_{3}$ & $P$ & PPC \\
\hline+105 & 28,3 & 31,3 & 53,3 & 0,4 & 0,03 & 1,6 & 28,2 & 28,8 & 16,1 & 20,7 & 24,2 \\
\hline$-105+74$ & 22,8 & 22,1 & 67,2 & 0,3 & 0,02 & 1,1 & 16,0 & 29,3 & 10,8 & 12,0 & 13,4 \\
\hline$-74+53$ & 20,3 & 26,4 & 60,4 & 0,4 & 0,03 & 1,3 & 17,0 & 23,4 & 13,5 & 15,5 & 14,1 \\
\hline$-53+38$ & 11,6 & 35,4 & 47,3 & 0,5 & 0,04 & 1,8 & 13,1 & 10,5 & 9,5 & 11,9 & 11,2 \\
\hline-38 & 17,0 & 47,6 & 24,8 & 1,9 & 0,08 & 4,1 & 25,7 & 8,0 & 50,1 & 39,9 & 37,2 \\
\hline A.R. & 100,0 & 31,5 & 52,4 & 0,6 & 0,03 & 1,9 & 100,0 & 100,0 & 100,0 & 100,0 & 100,0 \\
\hline Desl. & 89,0 & 30,8 & 53,3 & 0,5 & 0,03 & - & - & - & - & - & - \\
\hline Lama & 11,0 & 33,7 & 45,6 & 1,7 & 0,06 & - & - & - & - & - & - \\
\hline
\end{tabular}

A.R. - Alimentação recalculada. Fonte: Nascimento, 2010.

Os reagentes utilizados nos ensaios de flotação em bancada foram:

I. Depressor: meta-silicato de sódio (Vantec) - $\mathrm{Na}_{2} \mathrm{SiO}_{3} \cdot \mathrm{XH}_{2} \mathrm{O}$

II. Coletor: oleato de sódio $-\mathrm{CH}_{3}\left(\mathrm{CH}_{2}\right)_{7} \mathrm{CH}=\mathrm{CH}\left(\mathrm{CH}_{2}\right)_{7} \mathrm{COONa}$

III. Moduladores de $\mathrm{pH}: \mathrm{NaOH}$ e $\mathrm{HCl}$, ambos fabricados pela Vetec.

O preparo da solução de silicato de sódio $(0,5 \% \mathrm{p} / \mathrm{v})$ foi efetuado pela diluição do reagente em água destilada para a concentração desejada. No caso do oleato de sódio, efetuou-se a saponificação do mesmo com $\mathrm{NaOH}$, conforme metodologia descrita por Nascimento (2010).

Nos testes de flotação em escala de bancada (célula Cimag do Laboratório de Flotação do Departamento de Engenharia de Minas) foram analisadas as variáveis - deslamagem, porcentagem de sólidos, dosagem de coletor e dosagem de depressor - como fatores de influência para as

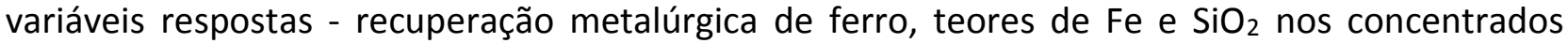
obtidos, usando o planejamento fatorial de experimentos de dois níveis, sem réplicas, conforme apresentado na Tabela 2. Tanto para o planejamento de experimentos quanto para a análise dos resultados dos ensaios de flotação foi utilizado o software estatístico Minitab 15.

Nesse trabalho estão apresentados os resultados dos ensaios de flotação direta em escala de bancada efetuados com uma amostra de minério de ferro do Quadrilátero Ferrífero-MG, utilizando oleato de sódio como coletor e meta-silicato de sódio como depressor em pH 9.

Tabela 2: Fatores e respectivos níveis para o planejamento fatorial da flotação.

\begin{tabular}{|c|c|c|c|}
\hline \multirow{2}{*}{ Fatores } & \multicolumn{2}{|c|}{ Níveis } & \multirow{2}{*}{ Legenda } \\
\cline { 2 - 3 } & - & + & \\
\hline Deslamagem & Sim & Não & A \\
\hline Porcentagem de sólidos (\%) & 30 & 60 & B \\
\hline Dosagem de oleato de sódio (g/t) & 600 & 1200 & C \\
\hline Dosagem de depressor $(\mathrm{g} / \mathrm{t})$ & 600 & 2400 & $\mathrm{D}$ \\
\hline
\end{tabular}

O procedimento padrão dos ensaios de flotação em bancada foi o seguinte:

I. adicionar o minério de ferro na cuba de $1 \mathrm{~L}$;

II. adicionar o volume de água necessário para obtenção da polpa com a porcentagem de sólidos desejada, descontando-se os volumes das soluções de oleato de sódio e de silicato de sódio; 
III. ajustar o rotor da célula de flotação para 1200 rpm;

IV. adicionar o volume de silicato de sódio (solução a $0,5 \% \mathrm{p} / \mathrm{v}$ ) correspondente à concentração desejada;

V. ajustar o valor de $\mathrm{pH}$ para 9 e condicionar por 6 minutos;

VI. adicionar o volume do oleato de sódio (solução a $1 \% \mathrm{p} / \mathrm{v}$ ) correspondente à concentração desejada e condicionar por 6 minutos, verificando-se o pH (se necessário, reajustar para 9);

VII. abrir a torneira de ar e flotar por 2 minutos;

VIII. retirar os produtos flotado e afundado, secá-los e pesá-los;

IX. após secagem e pesagem dos produtos flotado e afundado, homogeneizar, quartear e pulverizar para análise química.

\section{RESULTADOS E DISCUSSÃO}

$\mathrm{Na}$ Tabela 3 estão apresentados os resultados dos ensaios de flotação em bancada, conforme o planejamento estatístico. Pelos diagramas de Pareto, apresentados nas Figuras 1, 2 e 3 a porcentagem de sólidos, a deslamagem prévia do minério, a interação entre deslamagem do minério e dosagem de meta-silicato de sódio foram os fatores que foram significativos estatisticamente para recuperação de ferro. Para os teores de $\mathrm{Fe}$ e de $\mathrm{SiO}_{2}$ nos concentrados o único fator que mostrou ser significativo foi a porcentagem de sólidos.

Para melhor visualização dos resultados obtidos, nas Figuras 4, 5 e 6 estão apresentados os gráficos de cubo para os efeitos estudados (deslamagem, \% de sólidos, dosagem de metassilicato de sódio e dosagem de oleato de sódio) sobre as variáveis respostas: recuperação metalúrgica de $\mathrm{Fe}$, teores de $\mathrm{Fe}$ e $\mathrm{SiO}_{2}$ nos concentrados obtidos.

Tabela 3: Resultados obtidos dos testes do planejamento estatístico.

\begin{tabular}{|c|c|c|c|c|c|c|c|}
\hline Teste & A & B & C & $\mathbf{D}$ & $\mathbf{Y}_{\mathbf{1}}$ & $\mathbf{Y}_{\mathbf{2}}$ & $\mathbf{Y}_{\mathbf{3}}$ \\
\hline 1 & sim & 30 & 600 & 600 & 83,62 & 30,04 & 54,43 \\
\hline 2 & não & 30 & 600 & 600 & 71,9 & 31,7 & 51,89 \\
\hline 3 & sim & 60 & 600 & 600 & 59,84 & 42,79 & 35,59 \\
\hline 4 & não & 60 & 600 & 600 & 48,29 & 40,46 & 38,33 \\
\hline 5 & sim & 30 & 1200 & 600 & 80,2 & 33,87 & 48,82 \\
\hline 6 & não & 30 & 1200 & 600 & 66,81 & 28 & 57,43 \\
\hline 7 & sim & 60 & 1200 & 600 & 61,83 & 49,28 & 25,31 \\
\hline 8 & não & 60 & 1200 & 600 & 50,27 & 41,84 & 35,94 \\
\hline 9 & sim & 30 & 600 & 2400 & 79,37 & 30,02 & 54,65 \\
\hline 10 & não & 30 & 600 & 2400 & 81,83 & 31,58 & 52,21 \\
\hline 11 & sim & 60 & 600 & 2400 & 52,91 & 47,06 & 28,73 \\
\hline 12 & não & 60 & 600 & 2400 & 57,18 & 45,14 & 31,36 \\
\hline 13 & sim & 30 & 1200 & 2400 & 84,36 & 31,5 & 52,46 \\
\hline 14 & não & 30 & 1200 & 2400 & 73,05 & 32,65 & 50,33 \\
\hline 15 & sim & 60 & 1200 & 2400 & 50,82 & 47,18 & 28,48 \\
\hline 16 & não & 60 & 1200 & 2400 & 57,38 & 49,63 & 24,44 \\
\hline
\end{tabular}

$Y_{1}$ - Recuperação metalúrgica de Fe; $Y_{2}-$ Teor de Fe no concentrado e $Y_{3}$ - teor de $\mathrm{SiO}_{2}$ no concentrado 
Observa-se de uma maneira geral (Figuras 3, 4 e 5) que os teores de ferro foram maiores para maior \% de sólidos, o inverso ocorreu para teores de $\mathrm{SiO}_{2}$, que está coerente com a literatura (Huout, 1983; Lopes, 2009).

Pela análise de significância (Figuras 1, 2 e 3) e gráficos de cubo (Figuras 4, 5 e 6) ficou evidente a importância da deslamagem prévia do minério para eliminar as partículas menores do que $10 \mu \mathrm{m}$, que é a chave do processo de flotação aniônica de minério de ferro (Huout, 1983).

Nascimento (2010) e Nascimento et al. (2013) em estudos de microflotação com os minerais hematita e quartzo, verificaram a possibilidade do uso de silicato de sódio como depressor de quartzo para pequenas dosagens. Logo, poderia ser interessante a programação de novos experimentos para níveis menores de dosagem de silicato de sódio além da deslamagem para remoção das partículas de lama presentes no minério.

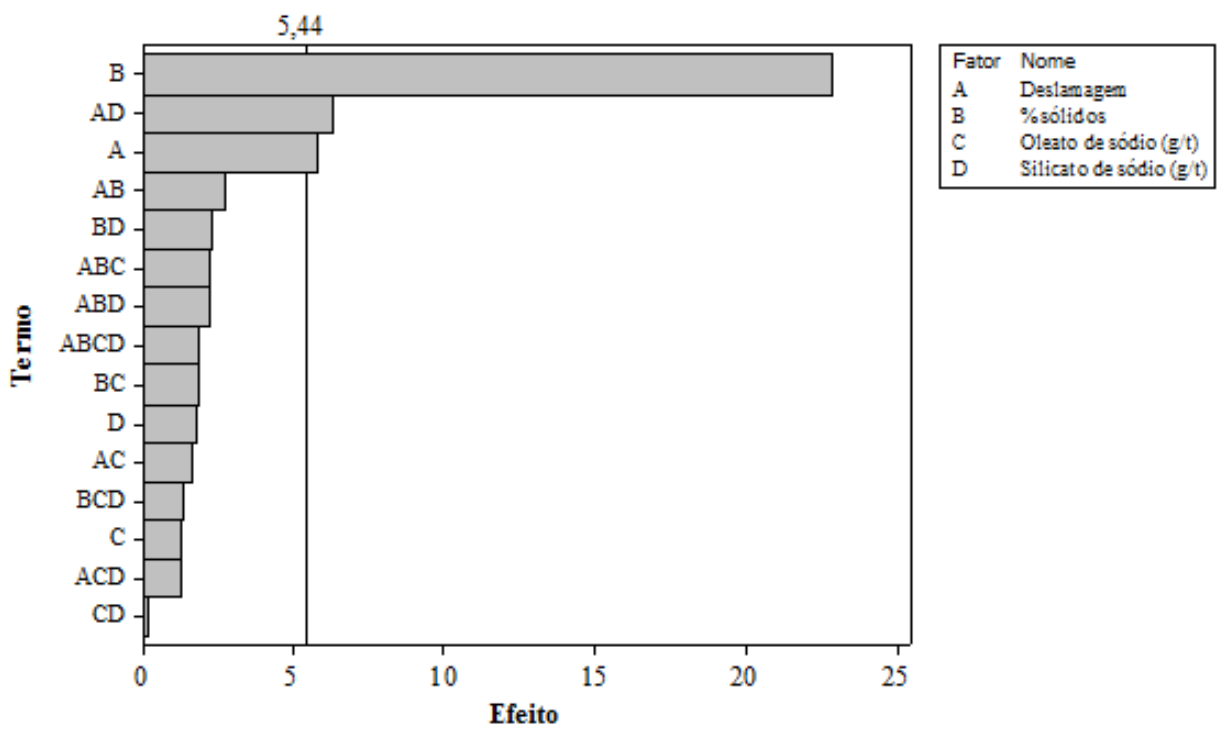

Lenth's PSE $=2,69813$

Figura 1: Gráfico Pareto para variável resposta recuperação metalúrgica de ferro $(a=0,10)$.

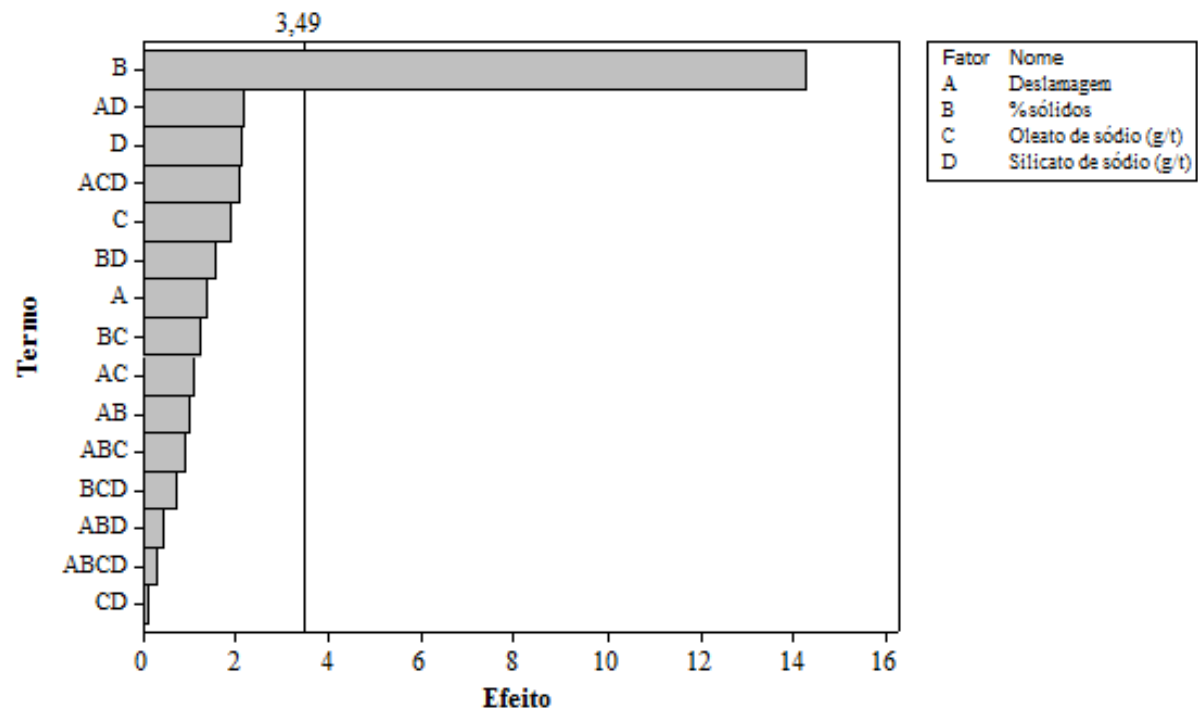

Lenth's PSE $=1,7325$

Figura 2: Gráfico Pareto para variável resposta teor de Fe no concentrado $(a=0,10)$. 


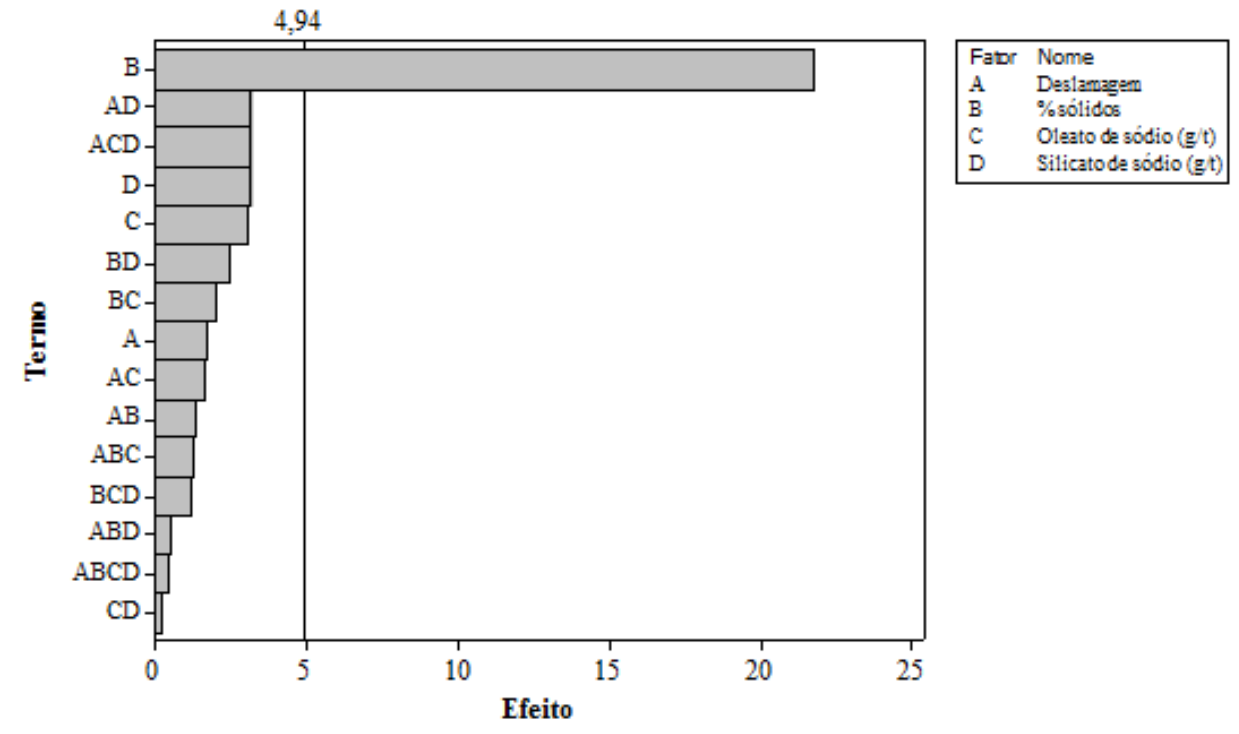

Lenth's PSE $=2,45062$

Figura 3: Gráfico Pareto para variável resposta teor de $\mathrm{SiO}_{2}$ no concentrado $(a=0,10)$.

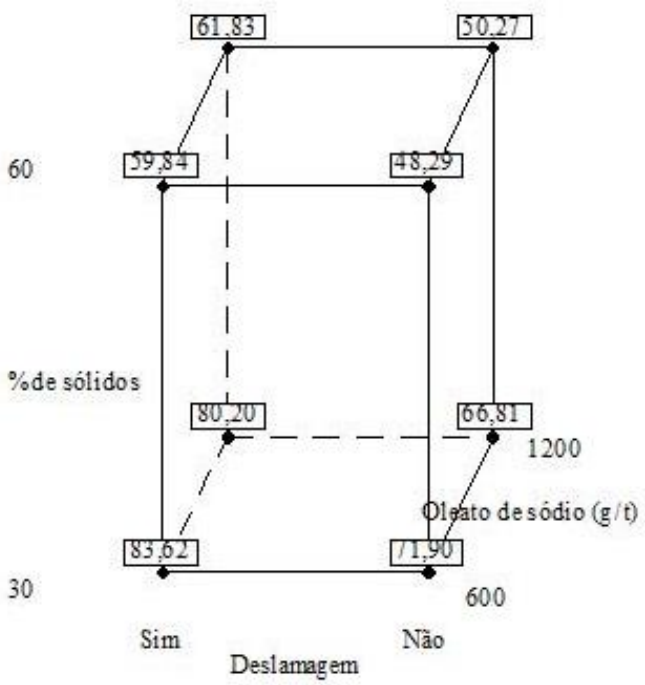

600

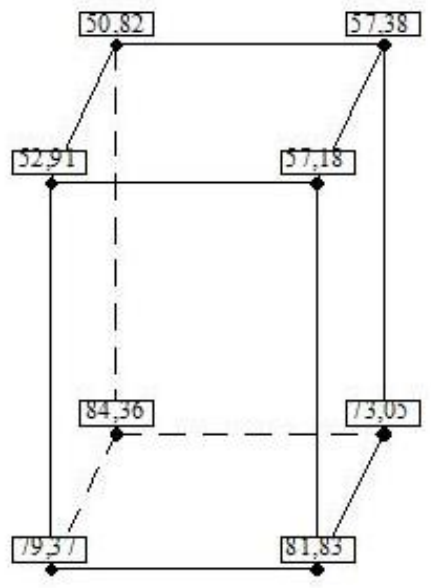

2400

Metassilicato de sódio $(\mathrm{g} / \mathrm{t})$

Figura 4 - Gráfico de cubos para recuperação metalúrgica de Fe. 


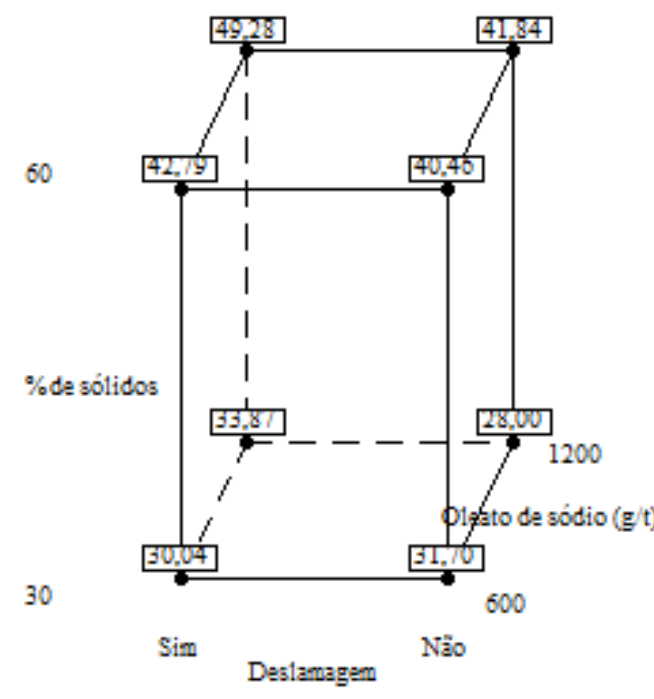

600

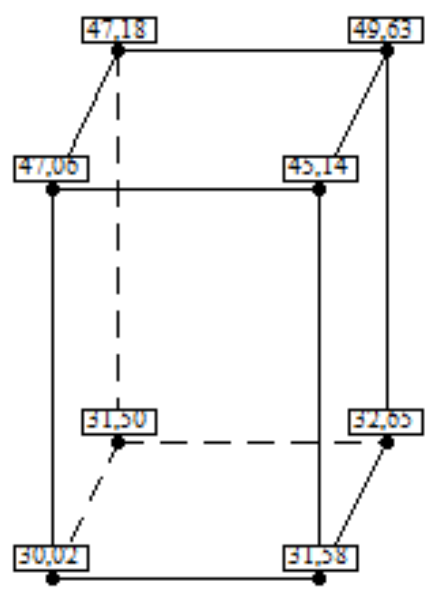

2400

Figure 5 - Gráfico de cubos para teor de Fe no concentrado.

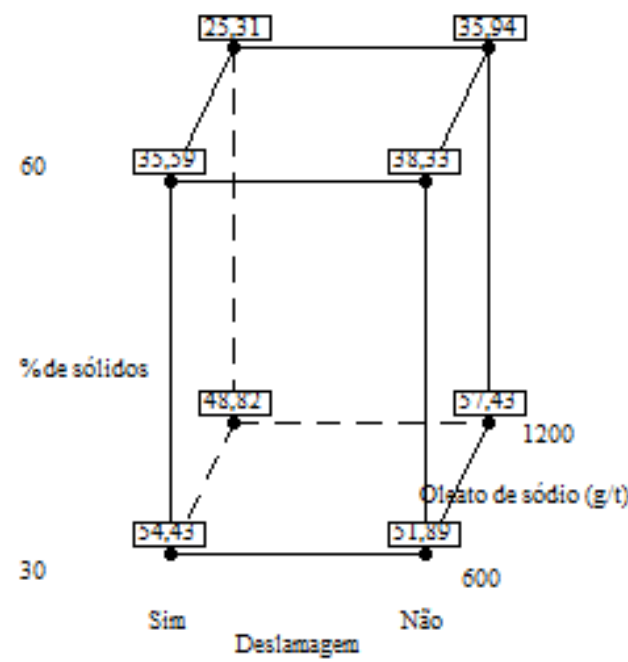

600

Metassilicato de sódio (g/t)

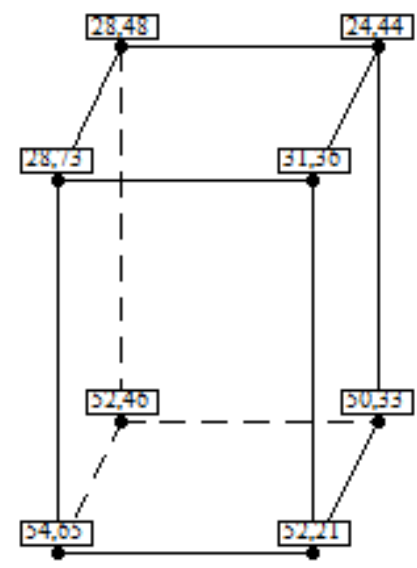

2400

Figura 6 - Gráfico de cubos para teor de Fe no concentrado.

\section{CONCLUSÕES}

Através dos resultados de flotação direta obtidos com amostra de minério de ferro em pH 9, a porcentagem de sólidos, a deslamagem prévia do minério, a interação entre deslamagem do minério e dosagem de meta-silicato de sódio foram os fatores que foram significativos estatisticamente para recuperação de ferro. Para os teores de $\mathrm{Fe}$ e de $\mathrm{SiO}_{2}$ nos concentrados o único fator que mostrou ser significativo foi a porcentagem de sólidos.

\section{AGRADECIMENTOS}

As autoras agradecem à Fapemig e a Vale pelo apoio financeiro, à CAPES e Fapemig pelas bolsas de mestrado e de iniciação científica, ao CNPq pela bolsa de produtividade em pesquisa. 


\section{REFERÊNCIAS}

1. ARAÚJO, A. C., AMARANTE, S. C., SOUZA C. C., SILVA, R. R. R. Ore mineralogy and its relevance for selection of concentration methods in processing of Brazilian iron ores. Mineral Processing and Extractive Metallurgy (Trans. Inst. Min. Metall. C). Vo.112,. pp. C44-C64 (2003).

2. ARAÚJO, A.C., VIANA, P.R., PERES, A. E. Reagents in iron ore flotation. Minerals Engeneering. Vol. 18. pp. 219-224 (2005).

3. HOUOT, R. Beneficiation of iron ore by flotation - review of industrial and potential applications. International Journal of Mineral Processing, 10(3), pp.182-204 (1983).

4. LIMA, R.M.F., LOPES, G. M., FRANCO, G. P. E COELHO P. A. Flotação de minérios de ferro de baixos teores. Relatório parcial. Laboratório de Propriedades Interfaciais. DEMIN. Universidade Federal de Ouro Preto. 73 p. (2008).

5. LOPES, G.M. , LIMA, R. M. F. Flotação direta de minério de ferro com oleato de sódio. REM: R. Esc. Minas, Vol. 62(3), pp. 323-329 (2009).

6. NASCIMENTO, D. R. Flotação aniônica de minério de ferro. Dissertação de Mestrado. PósGraduação em Engenharia Mineral. UFOP. Ouro Preto - MG, 115p. 2010.

7. NASCIMENTO, D. R., PEREIRA, R. D., LIMA, R. M. F. Influence of sodium silicate on floatability and charge of hematite and quartz with sodium oleate. Latin American Applied Research. 43:189-191 (2013). 